\title{
Fluorescence-guided Surgery with Splenic Preservation Prevents Tumor Recurrence in an Orthotopic Nude-mouse Model of Human Pancreatic Cancer
}

\author{
HO KYOUNG HWANG ${ }^{1,2,3}$, CHANG MOO KANG ${ }^{3}$, SUNG HWAN LEE ${ }^{3}$, TAKASHI MURAKAMI ${ }^{1,2,4}$, \\ TASUKU KIYUNA ${ }^{1,2,5}$, SE HOON KIM ${ }^{6}$, ROBERT M. HOFFMAN ${ }^{1,2}$ and MICHAEL BOUVET ${ }^{1,7}$ \\ ${ }^{1}$ Department of Surgery, University of California, San Diego, CA, U.S.A.; \\ ${ }^{2}$ AntiCancer, Inc., San Diego, CA, U.S.A.; \\ ${ }^{3}$ Department of Surgery, Division of Hepatobiliary and Pancreas Surgery, \\ Severance Hospital, Yonsei University College of Medicine, Seoul, Republic of Korea; \\ ${ }^{4}$ Graduate School of Medicine, Yokohama City University, Yokohama, Japan; \\ ${ }^{5}$ Department of Orthopedic Surgery, University of the Ryukyus, Okinawa, Japan; \\ ${ }^{6}$ Department of Pathology, Severance Hospital, Yonsei University College of Medicine, Seoul, Republic of Korea; \\ ${ }^{7}$ Veterans Affairs San Diego Healthcare System, San Diego, CA, U.S.A.
}

\begin{abstract}
Aim: The purpose of this study was to investigate whether splenectomy influences tumor recurrence after fluorescence-guided surgery $(F G S)$ in an orthotopic nudemouse model of pancreatic cancer. Materials and Methods: Green fluorescence protein (GFP)-labeled human pancreatic cancer cells (MiaPaCa2-GFP) were subcutaneously injected into the flanks of nude mice. Subcutaneous tumors were harvested and surgical orthotopic implantation (SOI) was performed in the tail of the pancreas with small tumor fragments. FGS was performed 21 days after SOI. Mice were then randomly divided into FGS-only control group $(n=7)$ and FGS plus splenectomy group $(n=8)$. Tumor recurrence was analyzed by laparotomy 21 days after FGS. Results: In the control group, no recurrence was found. In contrast, multiple peritoneal seeded nodules were observed in two mice of the splenectomy group (0\% vs. 25\%, p=0.467). Conclusion: Postoperative tumor recurrence only occurred in the splenectomy-treated group suggesting that FGS can spare the patient the morbidity of splenectomy.
\end{abstract}

This article is freely accessible online.

Correspondence to: Michael Bouvet, MD, Department of Surgery, University of California San Diego Moores Cancer Center, 3855 Health Sciences Drive, \#0987, La Jolla, CA 92093-0987 U.S.A. Tel: +18588226191, Fax: +1 8588226192,e-mail: mbouvet@ucsd.edu

Key Words: Fluorescence-guided surgery, splenectomy, pancreatic cancer, green fluorescent protein, recurrence, nude mice, orthotopic.
Pancreatic ductal adenocarcinoma (PDAC) is a lethal gastrointestinal malignant disease with overall 5-year survival rate of less than 5\% $(1,2)$. For left-sided PDAC, distal pancreatectomy including splenectomy has been accepted as standard procedure. The reason why splenectomy should be included is that margin-negative resection and effective regional lymph node clearance are possible through splenectomy. If the tumor is located away from the splenic hilum and there is no evidence of nodal metastasis around the spleen in preoperative imaging studies, splenectomy may not be necessary (3).

In some clinical studies, splenectomy had a negative effect on cancer survival of patients with gastric and colon cancer (4-6). In PDAC, splenectomy had a negative influence on long-term survival independent of disease-related factors after pancreatectomy for PDAC (7). We found that splenectomy enhanced tumor growth and peritoneal seeding in an orthotopic syngeneic murine pancreatic cancer model (8).

The purpose of the present study was to investigate whether simultaneous splenectomy influences the pattern of tumor recurrence when the primary tumor is completely resected with fluorescence-guided surgery (FGS) in an orthotopic human pancreatic cancer model.

\section{Materials and Methods}

Experimental design. Figure 1 shows the overall experimental design of this study. MiaPaCa2 green fluorescence protein (GFP)labeled cells $\left(2 \times 10^{6}\right)$ were injected subcutaneously into the right and left flanks of nude mice (Anticancer, Inc., San Diego, CA, USA). Subcutaneously-injected tumors grew to approximately $20 \mathrm{~mm}$ by 6 weeks after implantation. The tumors were then harvested for 


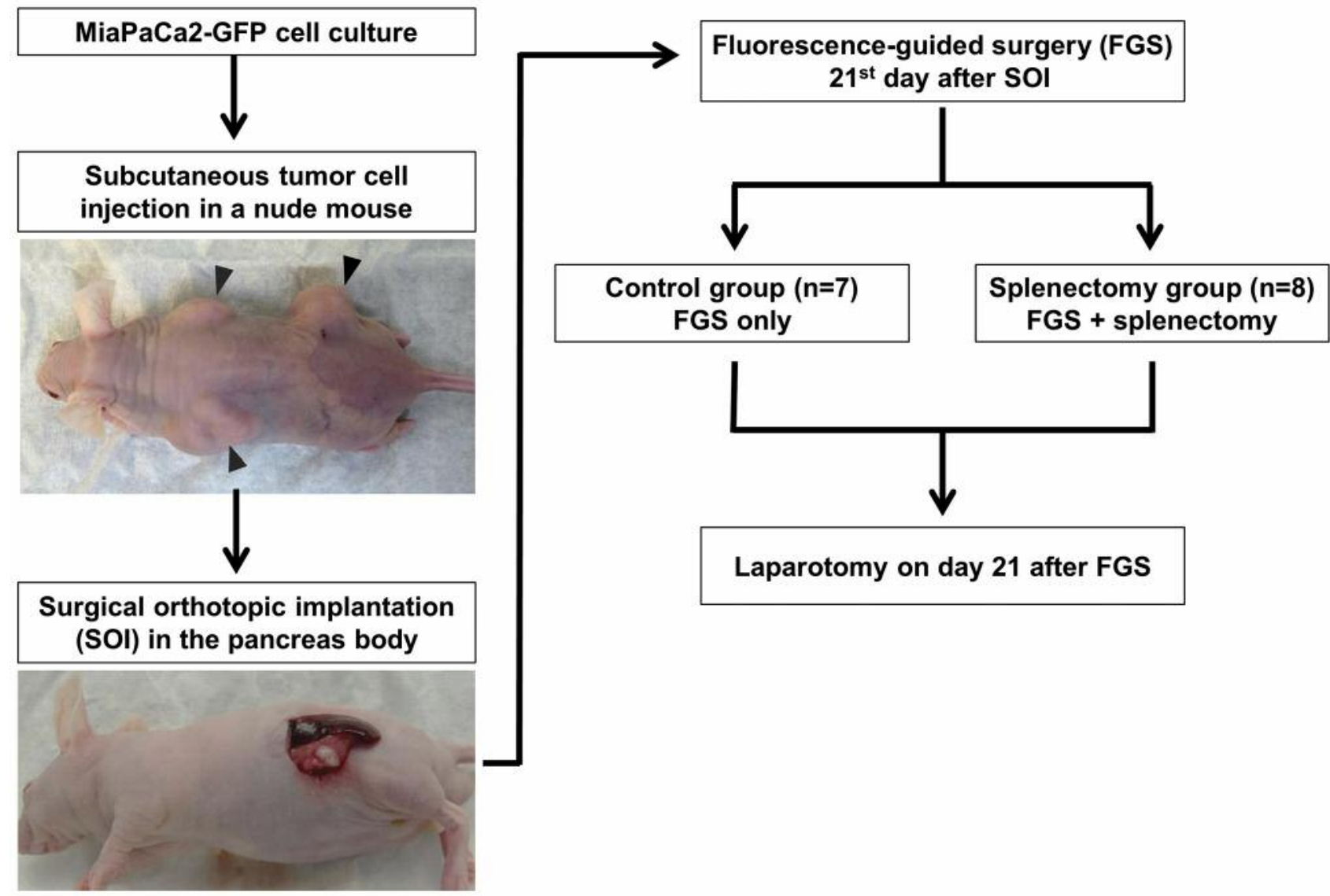

Figure 1. Schematic illustration for the experiment. MiaPaCa2-green fluorescent protein $(G F P)$ cells $\left(2 \times 10^{6}\right)$ were injected subcutaneously into the right and left flanks of nude mice. The subcutaneously implanted tumors grew to $20 \mathrm{~mm}$ by 6 weeks after implantation. The tumors were harvested for subsequent orthotopic implantation. A small fragment $\left(3 \mathrm{~mm}^{3}\right)$ was orthotopically implanted on the tail of the pancreas of the nude mice. Fluorescence-guided tumor resection was performed on day 21 after orthotopic tumor implantation. At this time, the mice were randomly divided into two groups according to splenectomy or not. Tumor recurrence was detected with laparotomy on day 21 after fluorescence-guided surgery (FGS).

subsequent orthotopic implantation. A small fragment $\left(3 \mathrm{~mm}^{3}\right)$ was orthotopically implanted on the tail of the pancreas of nude mice. Fluorescence-guided tumor resection was performed on day 21 after orthotopic tumor implantation. At this time, the mice were randomly divided into two groups according to splenectomy or not. Tumor recurrence was detected with laparotomy on day 21 after FGS.

Establishment of GFP-labeled cancer cell line. The MiaPaCa2 human pancreatic cell line was stably transfected with GFP as previously described $(9,10)$.

Cell culture. The MiaPaCa2-GFP human pancreatic cancer cells were maintained in Dulbecco's modified Eagle's medium (DMEM; Invitrogen, Carlsbad, CA, USA) supplemented with $10 \%$ fetal bovine serum (FBS; Sigma-Aldrich, St. Louis, MO, USA). The cells were incubated at $37^{\circ} \mathrm{C}$ in a humidified incubator with $5 \% \mathrm{CO}_{2}$ in air. The cells were collected after trypsinization and stained with trypan blue (Sigma-Aldrich, St. Louis, MO, USA). Only viable cells which excluded trypan blue were counted with a hemocytometer (Hausser Scientific, Horsham, PA, USA).
Mice. Athymic nu/nu nude mice, 4-6 weeks old, were used for subcutaneous cancer cell injection models and orthotopic tumor implant models. Mice were kept in a barrier facility under HEPA filtration and fed with autoclaved laboratory rodent diet. All mouse surgical procedures were performed with the animals anesthetized by intramuscular injection of a $0.02 \mathrm{ml}$ solution of $50 \%$ ketamine, $38 \%$ xylazine, and $12 \%$ acepromazine maleate. The animals were sacrificed on the 21 st day after surgery for investigating tumor recurrence. Intravenous injection of the ketamine mixture solution was used for euthanasia. To ensure death following injection, cervical dislocation was performed. All animal studies were conducted with an AntiCancer Institutional Animal Care and Use Committee protocol specifically approved for this study and in accordance with the principles and procedures outlined in the National Institute of Health Guide for the Care and Use of Animals under Assurance Number A3873-1.

Subcutaneous cancer cell injection. MiaPaCa2-GFP cells were harvested by trypsinization and washed twice with phosphatebuffered saline (PBS; Sigma Aldrich). The cells $\left(2 \times 10^{6}\right)$ were 


\section{Before FGS}
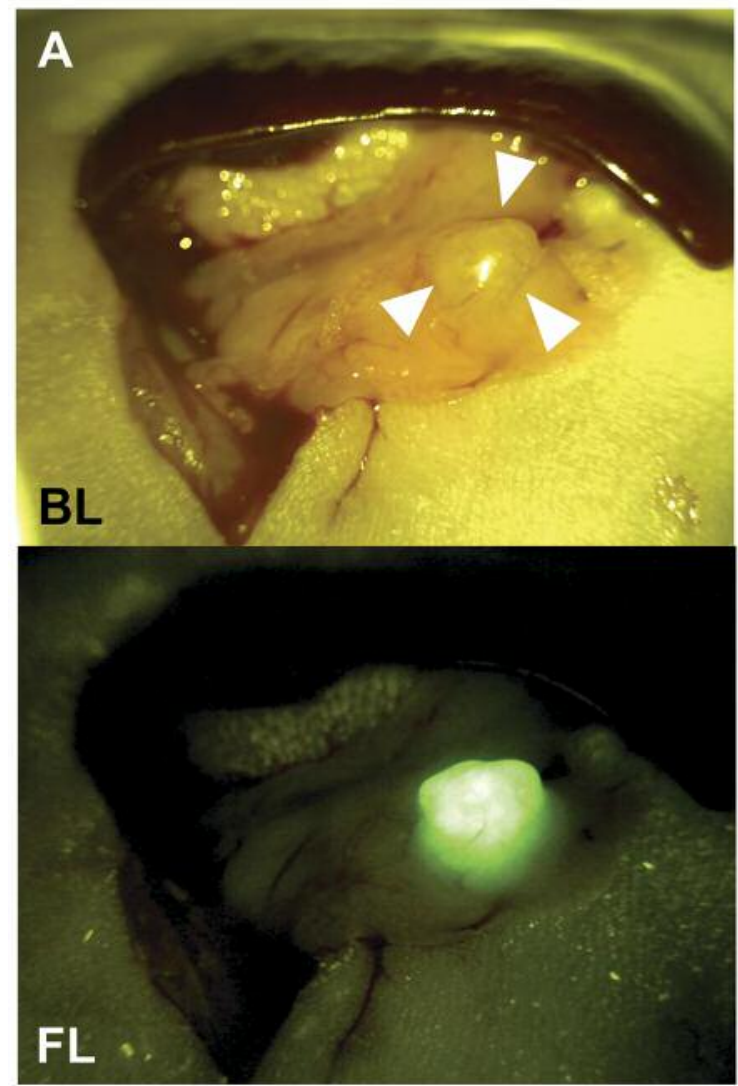

After FGS

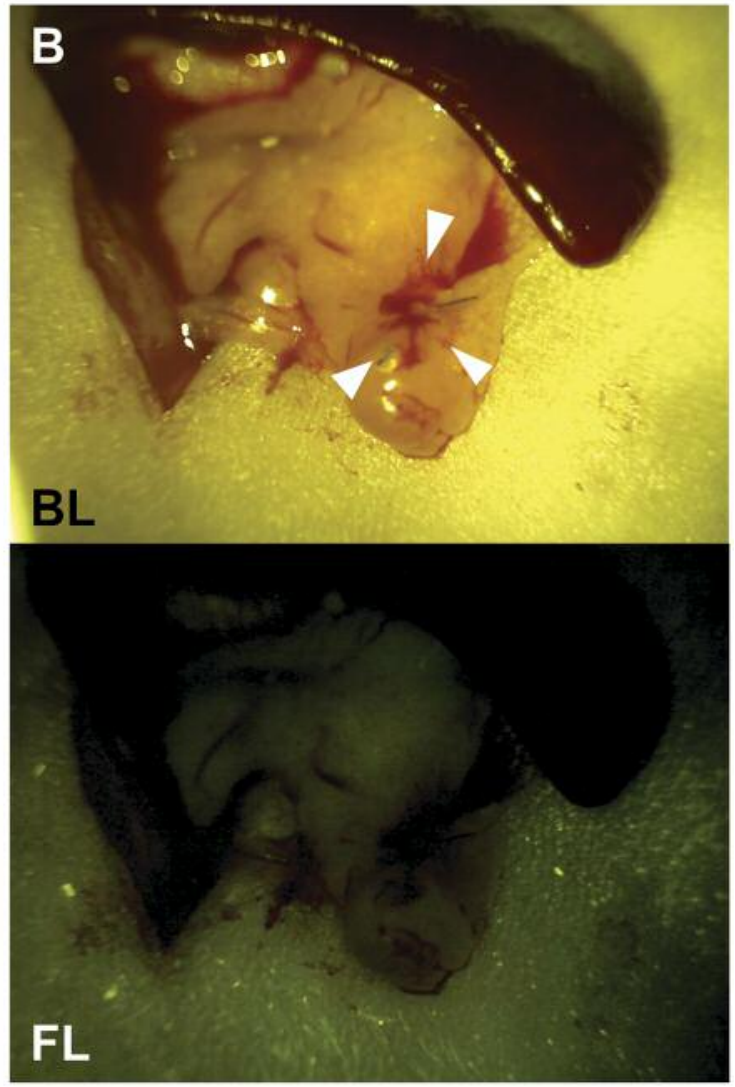

Figure 2. Fluorescence-guided surgery (FGS) was performed with a Dino-Lite system. Orthotopically-implanted tumors were identified by bright light $(B L)$ and fluorescence $(F L)$ before surgery $(A)$. After surgery $(B)$, the operative field showed no residual tumor.

injected subcutaneously into the right and left flanks of nude mice within $30 \mathrm{~min}$ of harvesting. Subcutaneously-injected tumors grew by about $20 \mathrm{~mm}$ at 6 weeks after implantation. The tumors were harvested for subsequent orthotopic implantation.

Orthotopic tumor implantation. The surgical orthotopic implantation (SOI) of tumor fragment was performed as previously described (11-13). A small 6-10 mm transverse incision was made on the left flank of the mouse through the skin and peritoneum. The pancreatic tail and spleen were exposed through this incision, and a single 3 $\mathrm{mm}$ tumor fragment retrieved from subcutaneous tumor of a nude mouse was sutured to the tail of the pancreas using 7-0 nylon surgical sutures (Dermalon ${ }^{\mathrm{TM}}$, Covidien; Medtronic Inc., MN, USA). The pancreatic tail and spleen were returned to the abdomen, and the incision was closed in one layer using 6-0 nylon surgical sutures $\left(\right.$ Dermalon $^{\mathrm{TM}}$ ).

Fluorescence-guided surgery. In order to achieve complete tumor resection, 3 weeks after SOI of MiaPaCa2-GFP to the pancreas, FGS was performed using a Dino-Lite imaging system (AM4113TGFBW Dino-Lite Premier; AnMo Electronics Corporation, Taiwan, ROC)
(14-16). When tumor resection was performed, the mice were randomly divided into two groups according to splenectomy or not. In the control group, a $10 \mathrm{~mm}$ transverse incision was made on the left flank, which was the same area during SOI of the mouse. The tail of the pancreas and spleen were exposed through this incision. The tumor was completely removed. The surgical resection field was carefully imaged with the Dino-Lite imaging system and the OV100 Small Animal Imaging System (Olympus, Tokyo, Japan) to detect microscopic residual tumor (16). The pancreatic tail and spleen were returned to the abdomen, and the incision was closed in one layer using 6-0 nylon surgical sutures (Dermalon ${ }^{\mathrm{TM}}$ ). In the splenectomy group, after tumor resection, the splenic artery and vein in the splenic hilum and short gastric vessels communicating with the splenic upper pole were securely ligated with 7-0 nylon suture $\left(\right.$ Dermalon $^{\mathrm{TM}}$ ), and the spleen was removed from the pancreas tail. Resected tumor length and width were measured with calipers and tumor volume was calculated by the following formula: tumor volume $=\left(\right.$ length $\mathrm{x}$ width $\left.{ }^{2}\right) / 2$.

Analysis of the tumor recurrence pattern. Tumor recurrence patterns were analyzed by laparotomy on day 21 after FGS. After 


\section{Control group}

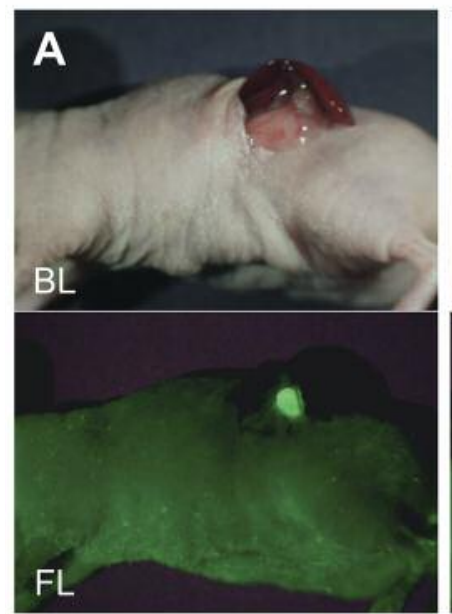

Before FGS

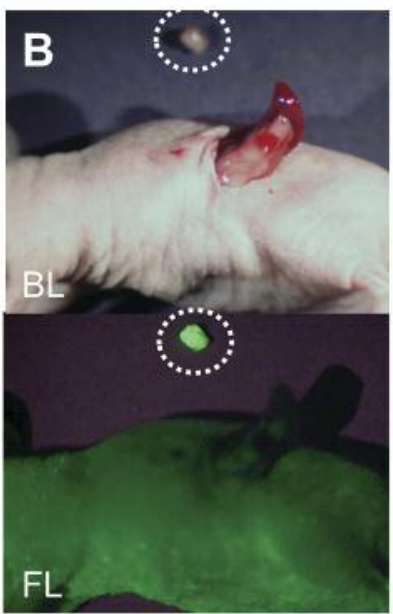

After FGS

\section{Splenectomy group}

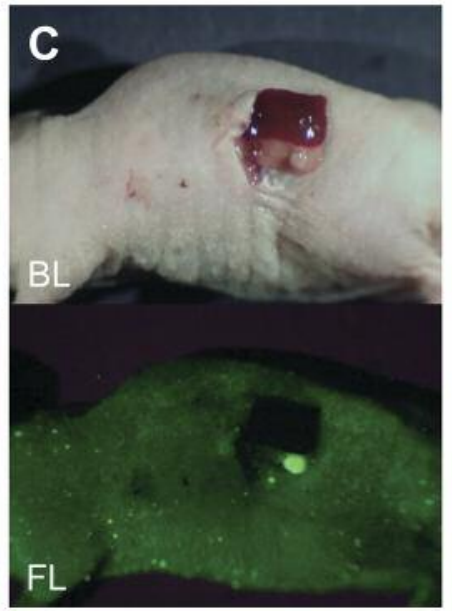

Before FGS

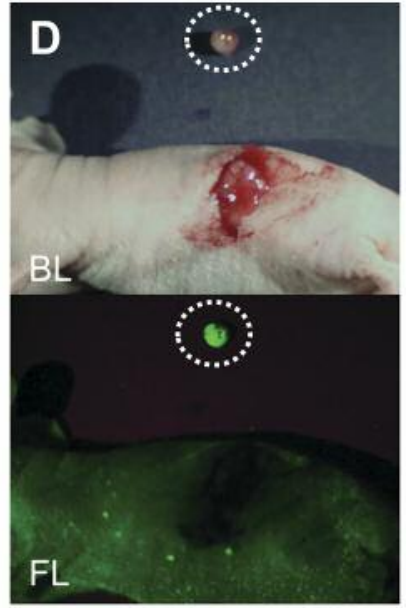

After FGS + splenectomy

Figure 3. Complete tumor resection after fluorescence-guided surgery $(F G S)$ was confirmed with the OV 100 imaging system. In the control group (A, $B)$, the operative field was observed with the OV 100 imaging system before (A) and after (B) FGS. In the splenectomy-treated group (C, D), FGS and splenectomy were simultaneously performed. The operative field was observed before $(C)$ and after $(D) F G S$ and splenectomy. There was no residual tumor on the pancreas in any mouse in the control and splenectomy group. BL: Bright light; FL: fluorescence. Dotted circles indicate the resected tumor visualized both in bright light and fluorescence.
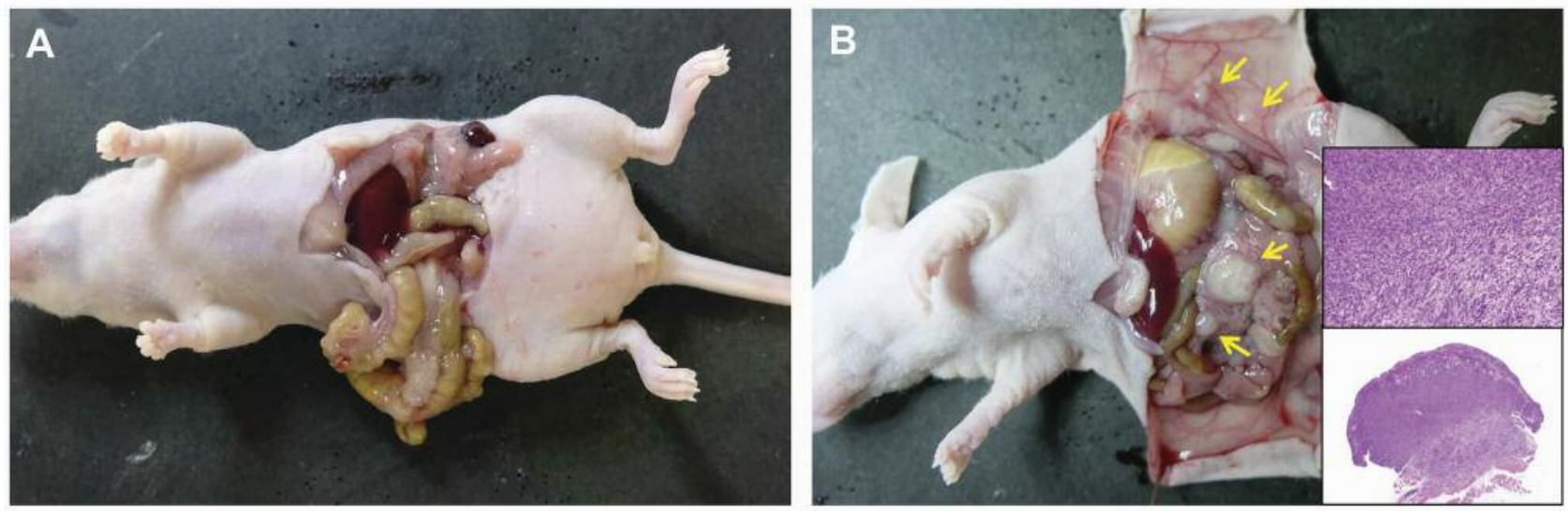

Figure 4. Tumor recurrence detection in control and splenectomy-treated groups. In the control group (A), no recurrence was found in any mouse. Multiple peritoneal recurrences (yellow arrows) were observed in two mice in the splenectomy-treated group (B). Seeded nodules were confirmed as recurred pancreatic cancer by histological analysis (insets).

laparotomy, the peritoneal cavity was carefully assessed for the evidence of peritoneal, hepatic, or other site metastases.

Histology. The fresh resected tumors were fixed in $10 \%$ formalin, and then embedded in paraffin. The tissue sections, at a thickness of 4- $\mu \mathrm{m}$, were deparaffinized in xylene and rehydrated in an ethanol series. Hematoxylin and eosin staining was performed according to standard protocols for histological confirmation of tumor.

Statistical analysis. All statistical analyses were performed with SPSS 20.0 software (IBM, Armonk, NY, USA). Categorical variables were compared using chi-squared or Fisher exact tests. Continuous variables are presented as the mean \pm standard deviation $(\mathrm{SD})$ and the significance of differences was determined using Student's $t$-test. A $p$-value of 0.05 or less indicated statistical significance.

\section{Results}

FGS without splenectomy was performed in seven mice, and FGS plus splenectomy was performed in eight mice. Mean body weight at SOI, FGS and laparotomy was not significantly different between the two groups. Mean 
Table I. General characteristics and tumor recurrence pattern in mice. Data are the mean \pm SD number (\%) of mice.

\begin{tabular}{lccc}
\hline Characteristic & $\begin{array}{c}\text { Control } \\
\text { group }(\mathrm{n}=7)\end{array}$ & $\begin{array}{c}\text { Splenectomy } \\
\text { group (n=8) }\end{array}$ & $p$-Value \\
\hline Body weight, g & & & \\
$\quad$ At SOI & $19.5 \pm 1.3$ & $20.8 \pm 1.1$ & 0.070 \\
At tumor resection & $22.1 \pm 1.1$ & $23.2 \pm 1.0$ & 0.090 \\
At laparotomy & $29.2 \pm 2.1$ & $30.3 \pm 0.9$ & 0.239 \\
Tumor dimensions & & & 0.640 \\
Length, mm & $3.2 \pm 0.7$ & $3.0 \pm 0.9$ & 0.450 \\
Width, mm & $2.5 \pm 0.6$ & $2.2 \pm 0.7$ & 0.570 \\
Volume, mm & & $9.5 \pm 8.6$ & 0.467 \\
Tumor recurrence & $11.9 \pm 6.1$ & $6(75 \%)$ & \\
No & $7(100 \%)$ & $2(25 \%)$ & \\
Yes & 0 & & \\
\hline
\end{tabular}

SOI: Surgical orthotopic implantation.

resected tumor length, width and total tumor volume were not significantly different between the two groups (Table I). After FGS, the Dino-Lite and OV100 imaging systems detected no residual tumor in the operative fields in either group (Figures 2 and 3).

Tumor recurrence patterns. In the non-splenectomy group, no recurrence was found in any mouse. Multiple peritoneal seeded nodules were observed in two mice $(25 \%)$ of the splenectomy group (Table I). Seeded nodules were confirmed as recurrent pancreatic cancer by histological analysis (Figure 4). There were no hepatic or other metastases.

\section{Discussion}

The exact mechanism of tumor suppression that the spleen is responsible for remains uncertain. One of the possible mechanisms is that the activity of natural killer (NK) cells can decrease after splenectomy. Imai et al. reported the number of pulmonary metastases in splenectomized mice was significantly greater than in the control group and they suggested that splenic NK cells play an important role in the suppression of pulmonary metastasis in the mouse liver tumor model (17).

Another possible mechanism is that regulatory T-cells (Tregs) [cluster of differentiation $4\left(\mathrm{CD} 4^{+}\right) \mathrm{CD} 25^{+}$forkhead box P3 $\left.\left(\mathrm{FOXP}^{+}\right)\right]$may increase after splenectomy. Tregs play a role in immunological tolerance to self-antigen and in suppression of antitumor immunity (18). High FOXP3 ${ }^{+}$Treg infiltration was significantly associated with shorter overall survival in the majority of patients with solid tumors (19). Higashijima et al. reported that the number of hepatic metastases significantly increased in the splenectomy group compared to the spleen-preserved group after colon cancer cells were injected into the spleen of mice. Their conclusion was that splenectomy enhanced hepatic metastasis through the increase of Foxp3 mRNA in the liver (20).

In our previous clinical study to evaluate the prognostic impact of tumor-infiltrating lymphocytes in resected leftsided PDAC, we demonstrated that a higher ratio of cytotoxic T-cells to Tregs was significantly related to better survival in patients with PDAC (21). In another previous study, we also found that the tumor volume was significantly larger and peritoneal seeding occurred more frequently in the splenectomy group in an orthotopic syngeneic murine pancreatic cancer model (8). In that previous study, the ratios of tumor-infiltrating $\mathrm{CD}^{+}$cells and $\mathrm{CD}^{+}$to $\mathrm{FOXP}^{+}$cells were significantly higher in the control group compared to the splenectomy group.

In the present study, the possible mechanism for multiple peritoneal seeding in the splenectomy group may be due to the decreased activity of splenic NK cells, since we used athymic nude mice as the hosts. Future experiments will compare NK cells in the two groups. Chemokines will also be investigated in the splenectomy and spleen-preserved groups (22). In conclusion, we found that multiple peritoneal seeding occurred only in the splenectomy group after fluorescence-guided pancreatic cancer resection.

\section{Funding}

This study was supported by a faculty research grant of Yonsei University College of Medicine for 2015 (6-2015-0162) and by grants from the National Cancer Institute CA142669 and CA132971 (to M.B. and AntiCancer, Inc) and VA Merit Review Award 1 I01 BX003856-01A1 (to M.B.).

\section{References}

1 Hidalgo M: Pancreatic cancer. N Engl J Med 362(17): 16051617,2010

2 Li D, Xie K, Wolff R and Abbruzzese JL: Pancreatic cancer. Lancet 363(9414): 1049-1057, 2004.

3 Kim SH, Kang CM, Satoi S, Sho M, Nakamura Y and Lee WJ: Proposal for splenectomy-omitting radical distal pancreatectomy in well-selected left-sided pancreatic cancer: Multicenter survey study. J Hepatobiliary Pancreat Sci 20(3): 375-381, 2013.

4 Davis CJ, Ilstrup DM and Pemberton JH: Influence of splenectomy on survival rate of patients with colorectal cancer. Am J Surg 155(1): 173-179, 1988.

5 Griffith JP, Sue-Ling HM, Martin I, Dixon MF, McMahon MJ, Axon AT and Johnston D: Preservation of the spleen improves survival after radical surgery for gastric cancer. Gut 36(5): 684690, 1995.

6 Wanebo HJ, Kennedy BJ, Winchester DP, Stewart AK and Fremgen AM: Role of splenectomy in gastric cancer surgery: Adverse effect of elective splenectomy on longterm survival. J Am Coll Surg 185(2): 177-184, 1997.

7 Schwarz RE, Harrison LE, Conlon KC, Klimstra DS and Brennan MF: The impact of splenectomy on outcomes after resection of pancreatic adenocarcinoma. J Am Coll Surg 188(5): 516-521, 1999. 
8 Hwang HK, Murakami T, Kiyuna T, Kim SH, Lee SH, Kang CM, Hoffman RM and Bouvet M: Splenectomy is associated with an aggressive tumor growth pattern and altered host immunity in an orthotopic syngeneic murine pancreatic cancer model. Oncotarget 8(51): 88827-88834, 2017.

9 Bouvet M, Tsuji K, Yang M, Jiang P, Moossa AR and Hoffman $\mathrm{RM}$ : In vivo color-coded imaging of the interaction of colon cancer cells and splenocytes in the formation of liver metastases. Cancer Res 66(23): 11293-11297, 2006.

10 Katz MH, Takimoto S, Spivack D, Moossa AR, Hoffman RM and Bouvet $\mathrm{M}$ : A novel red fluorescent protein orthotopic pancreatic cancer model for the preclinical evaluation of chemotherapeutics. J Surg Res 113(1): 151-160, 2003.

11 Bouvet M, Wang J, Nardin SR, Nassirpour R, Yang M, Baranov E, Jiang P, Moossa AR and Hoffman RM: Real-time optical imaging of primary tumor growth and multiple metastatic events in a pancreatic cancer orthotopic model. Cancer Res 62(5): 1534-1540, 2002.

$12 \mathrm{Fu} \mathrm{X}$, Guadagni F and Hoffman RM: A metastatic nude-mouse model of human pancreatic cancer constructed orthotopically with histologically intact patient specimens. Proc Natl Acad Sci USA 89(12): 5645-5649, 1992.

13 Furukawa T, Kubota T, Watanabe M, Kitajima M and Hoffman RM: A novel "patient-like" treatment model of human pancreatic cancer constructed using orthotopic transplantation of histologically intact human tumor tissue in nude mice. Cancer Res 53(13): 3070-3072, 1993.

14 Hiroshima Y, Maawy A, Metildi CA, Zhang Y, Uehara F, Miwa S, Yano S, Sato S, Murakami T, Momiyama M, Chishima T, Tanaka K, Bouvet M, Endo I and Hoffman RM: Successful fluorescence-guided surgery on human colon cancer patientderived orthotopic xenograft mouse models using a fluorophoreconjugated anti-CEA antibody and a portable imaging system. J Laparoendosc Adv Surg Tech A 24(4): 241-247, 2014.

15 Hiroshima Y, Maawy A, Sato S, Murakami T, Uehara F, Miwa S, Yano S, Momiyama M, Chishima T, Tanaka K, Bouvet M, Endo I and Hoffman RM: Hand-held high-resolution fluorescence imaging system for fluorescence-guided surgery of patient and cell-line pancreatic tumors growing orthotopically in nude mice. J Surg Res 187(2): 510-517, 2014.
16 Hiroshima Y, Maawy A, Zhang Y, Sato S, Murakami T, Yamamoto M, Uehara F, Miwa S, Yano S, Momiyama M, Chishima T, Tanaka K, Bouvet M, Endo I and Hoffman RM: Fluorescence-guided surgery in combination with UVC irradiation cures metastatic human pancreatic cancer in orthotopic mouse models. PLoS One 9(6): e99977, 2014.

17 Imai S, Nio Y, Shiraishi T, Tsubono M, Morimoto H, Tseng CC, Kawabata K, Masai Y and Tobe T: Effects of splenectomy on pulmonary metastasis and growth of SC42 carcinoma transplanted into mouse liver. J Surg Oncol 47(3): 178-187, 1991.

18 Yagi H, Nomura T, Nakamura K, Yamazaki S, Kitawaki T, Hori S, Maeda M, Onodera M, Uchiyama T, Fujii S and Sakaguchi S: Crucial role of FOXP3 in the development and function of human $\mathrm{CD} 25^{+} \mathrm{CD} 4{ }^{+}$regulatory T-cells. Int Immunol 16(11): 1643-1656, 2004.

19 Shang B, Liu Y, Jiang SJ and Liu Y: Prognostic value of tumorinfiltrating foxp $3^{+}$regulatory $\mathrm{t}$ cells in cancers: A systematic review and meta-analysis. Sci Rep 5: 15179, 2015.

20 Higashijima J, Shimada M, Chikakiyo M, Miyatani T, Yoshikawa K, Nishioka M, Iwata T and Kurita N: Effect of splenectomy on antitumor immune system in mice. Anticancer Res 29(1): 385-393, 2009.

21 Hwang HK, Kim HI, Kim SH, Choi J, Kang CM, Kim KS and Lee WJ: Prognostic impact of the tumor-infiltrating regulatory T-cell (Foxp3+)/activated cytotoxic T lymphocyte (granzyme $\mathrm{B}+)$ ratio on resected left-sided pancreatic cancer. Oncol Lett 12(6): 4477-4484, 2016.

22 Matsuo Y, Raimondo M, Woodward TA, Wallace MB, Gill KR, Tong Z, Burdick MD, Yang Z, Strieter RM, Hoffman RM and Guha S: CXC-chemokine/CXCR2 biological axis promotes angiogenesis in vitro and in vivo in pancreatic cancer. Int $\mathrm{J}$ Cancer 125(5): 1027-1037, 2009.
Received November 7, 2017

Revised November 25, 2017

Accepted November 28, 2017 